\title{
Implementation of real time auto focus methods for static and dynamic infrared imaging of power semiconductor chips
}

\author{
by Daniela Florian ${ }^{\star * * *}$, Sascha Einspieler ${ }^{\star, * *}$, Helmut Köck ${ }^{*, * *}$, Michael Glavanovics ${ }^{\star}$, Daniel Wischounig-Strucl ${ }^{* \star}$
}

“KAI (Kompetenzzentrum Automobil- und Industrieelektronik) GmbH, Europastrasse 8, 9524 Villach, Austria, daniela.florian@k-ai.at; sascha.einspieler@k-ai.at; helmut.koeck@k-ai.at; michael.glavanovics@k-ai.at

*Institute of Networked and Embedded Systems, Alpen-Adria-Universität Klagenfurt, Universitätstr. 65-67, 9020 Klagenfurt, AustriaDaniel.Wischounig-Strucl@aau.at

\begin{abstract}
The implementation of an auto focus algorithm is an important and crucial feature for infrared microscopy. To detect focus images, the entire infrared camera has to be moved due to the fact that a microscope camera system employs a fixed lens.

In our work, we present the concept of a real time auto focus algorithm especially for infrared imaging of power semiconductor chips. Three auto focus functions are implemented and compared: the gradient operator, the Sobel edge detection and the 2D Fast Fourier Transformation (FFT). The main goal is to identify the function which can robustly detect the focus image on varying temperatures and at changing intensities.
\end{abstract}

\section{Introduction}

During the infrared (IR) measurements the target system starts moving due to the thermal expansion; hence the focus has to be adjusted either manually or automatically [1]. In literature, two methods to detect the focus images are described, active focusing and passive focusing. In active focusing, the distance to the object is measured and the system is adjusted for an accurate focus. In passive focusing, the content of the image is analyzed. For each focus position in the optical axis a characteristic value, called focus value, is recorded and stored in the focus curve. The focus position is detected by searching for the maximum of the focus curve [2].

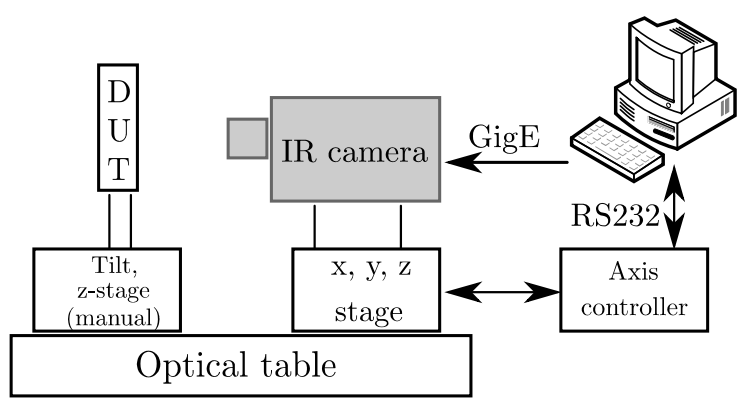

Fig. 1. Infrared thermography system

The thermal characterization measurement setup applied for this paper consists of an electrical stress system, an IR thermography system and additional measurement and control components, like oscilloscope and trigger systems. The IR thermography system contains an IR camera model Thermosensorik InSb640SM, remote controlled positioning stages and a device under test (DUT) mounting unit (see figure 1). The remote controlled positioning stage in $x$ direction (optical axis) operates over a range from $-50 \mathrm{~mm}$ to $50 \mathrm{~mm}$ and has a maximal spatial resolution of $1 \mu \mathrm{m}$. The expected focus image lies in the range of $-50 \mathrm{~mm}$ to $0 \mathrm{~mm}$. Typically, the range $0 \mathrm{~mm}$ to $50 \mathrm{~mm}$ is used as parking position for the IR camera.

Table 1. Specification of the IR camera

\begin{tabular}{|l|l|}
\hline Item & Values \\
\hline Image format & $640 \times 512$ pixel \\
\hline Pixel pitch & $15 \mu \mathrm{m}$ \\
\hline Image resolution & $14 \mathrm{bit}$ \\
\hline Maximum full-frame rate & $100 \mathrm{~Hz}$ \\
\hline Detector material & Indium Antimonide \\
\hline
\end{tabular}

The specification of the IR camera is described in table 1. The IR camera can be equipped with a fixed standard macro lens or a $2.5 x$ magnification microscope lens. Due to the fixed position of the lens relative to the IR detector, the entire camera system has to be moved in order to detect the focus image and the corresponding focus position. Further 
information about this IR camera and setup can be found in [3] and [4]. In the current paper, the implementation and evaluation of three real time auto focus functions are presented. Then the main challenges of these functions especially for IR thermography are discussed.

\section{Auto focus functions and methods}

The basic concept of the auto focus function is explained referring to the flowchart of figure 2 . The auto focus function contains one search loop with four search cases. For each search loop initial and ending values are defined, wherein the expected focus position is located. Within this search range, the focus curve is recorded. The focus value $Y_{F}$ is computed as

$$
\Upsilon_{F}=\sum_{x=1}^{h} \sum_{y=1}^{w} \Gamma\left\{\begin{array}{c}
I, \text { edge } \\
I, 2 D F F T \\
I, \text { gradient }
\end{array},\right.
$$

where $\mathrm{I}$ is the current input image, $\Gamma$ represents the auto focus function, $\mathrm{h}$ and $\mathrm{w}$ are the dimension of the image. The focus value is computed for each position with a step size, depending on the selected auto focus function. As auto focus functions, the Sobel edge detector [5], the 2D FFT [6] and the gradient operator are applied.

For each method we define a hypothesis, how the auto focus method can determine the focus image. The gradient operator in combination with a filter method (like Sobel) and a threshold function is used to detect edges in the images. Consequently, when using edge detection, focus images show more edges than blurred ones. To obtain local intensity changes, the gradient operator is applied to each pixel of the image together with its nearest neighbors. To cover horizontal and vertical edge structures in the image, the gradient operator is calculated in $\mathrm{X}$ and $\mathrm{Y}$ direction. Thus, the basic hypothesis for the gradient operator is that the intensity changes in focus images are greater than in blurred images. A common image processing method to filter images is the 2D FFT. The image is transformed to the frequency domain. A particular frequency is represented by each point in the spatial frequency domain. Typically, sharp images contain more high spatial frequencies in their spectrum than blurred images. For the case of the 2D FFT method, focus images are identified by the amount of high frequency components.

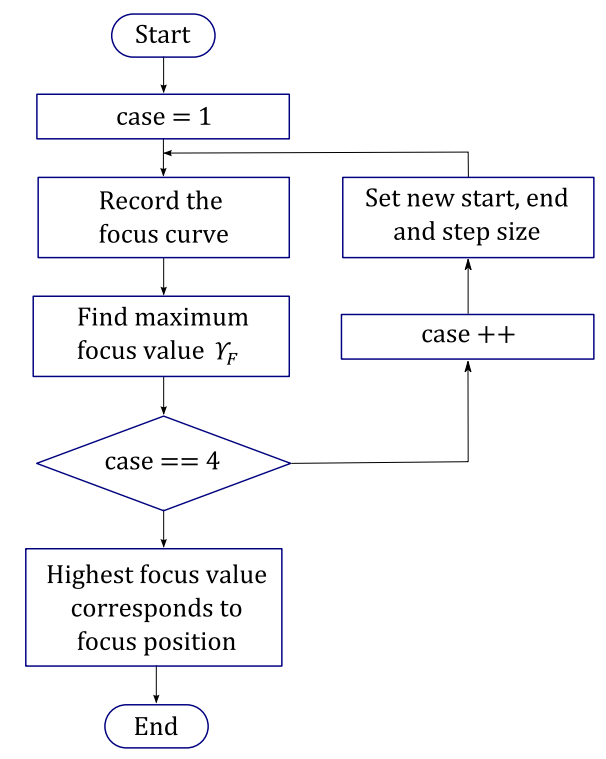

Fig. 2. Flowchart of the auto focus algorithm

Figure 3 shows an example of the search loop execution. All curves are normalized to the same scale. For the first case a rough step size and large search range are implemented. In the next step the maximum in the focus curve is detected and the position of the maximum is referred to the center position of the next case. In this example the highest focus value is detected at position $-48 \mathrm{~mm}$ in the first case. For consecutive cases the step size and search range are narrowed. For the second case a step size of $0.1 \mathrm{~mm}$ is selected and the focus curve is recorded. In this case the focus position is detected at $-47.9 \mathrm{~mm}$. In the third case the step size is reduced to $0.01 \mathrm{~mm}$ and the focus value is near to the maximum. In the last case a fine step size of $0.005 \mathrm{~mm}$ is used to detect the correct focus image and the corresponding focus position (see figure 3 - Case 4). Four positions are recorded and evaluated. The evaluation shows that a stage resolution of $0.005 \mathrm{~mm}$ is adequate to detect the desired focus image, hence a smaller step size $(<0.005 \mathrm{~mm})$ is not useful. For this example the focus position is detected at position $-47.955 \mathrm{~mm}$. 
The evaluation shows that four cases with the selected search ranges and step sizes are adequate to detect the desired focus image and corresponding focus positions. In general, it is possible to adjust and optimize each case for the given application.

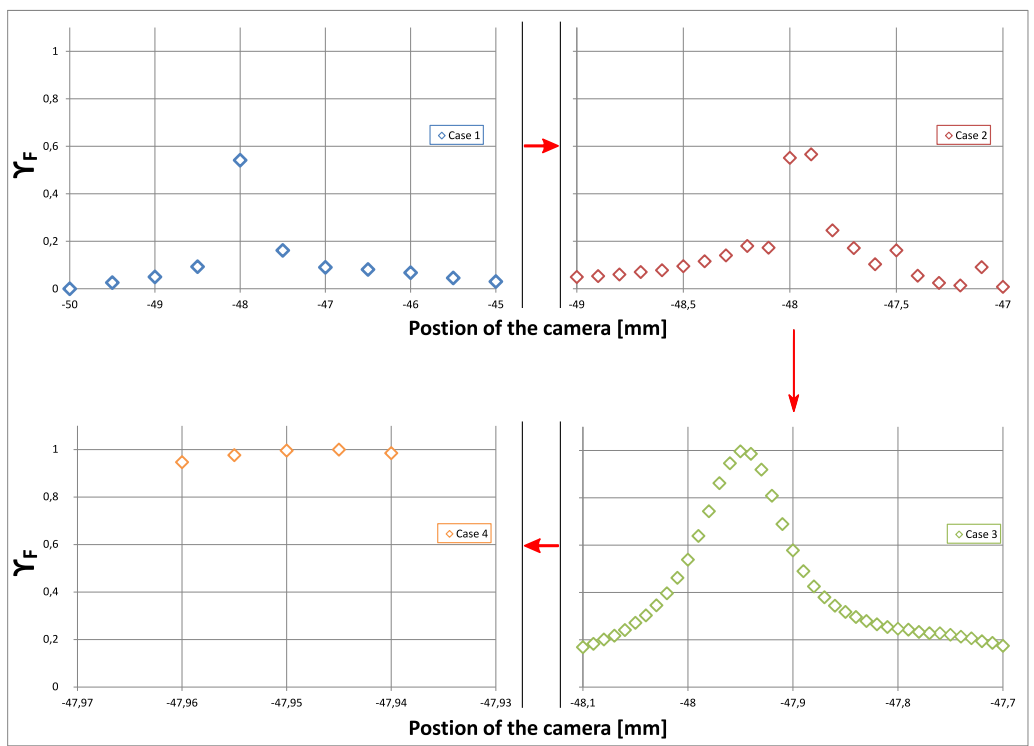

Fig. 3. Example of the search cases. All curves are normalized between 0 and 1. The focus position is detected, if the focus value reaches the maximum.

\section{Results}

For the evaluation three power semiconductor DUTs with significantly different structures are applied. Two of these (marked by T26 and E62) belong to the smart power switch family [7] and the third one (marked by m102) is a test device without logic structures, showing only simple structures on the surface. Photographs of the test chips are given in figure 4.

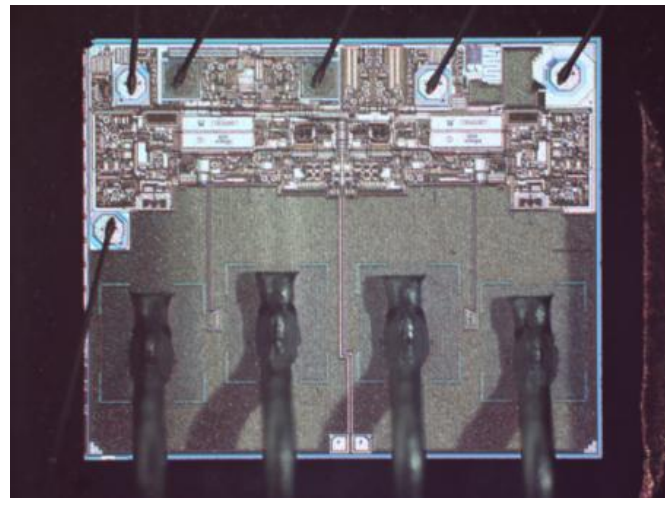

a) Device T26

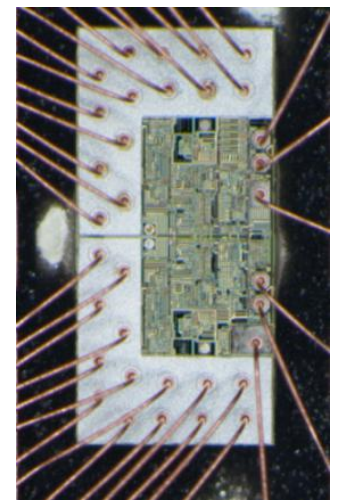

b) Device E62

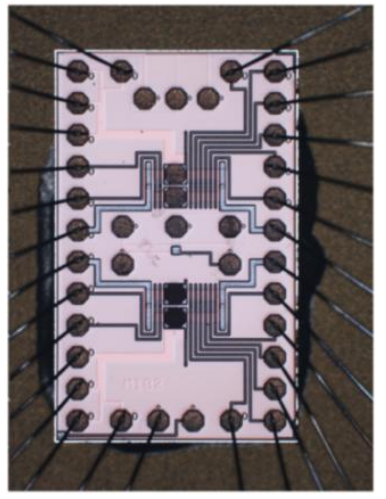

c) Device m102

Fig. 4. Optical photographs of partially opened power semiconductors

\subsection{Visual evaluation of the focus image}

For the evaluation an Intel ${ }^{\circledR}$ Core ${ }^{T M} 2$ Duo $\mathrm{CPU}$ with $2.33 \mathrm{GHz}$ and $2 \mathrm{~GB}$ of RAM is used. Matlab $2009 \mathrm{~b}$ serves as development and application software platform. We are working with the raw data values of the IR camera. The analog to digital converter provides pixel data over a range of 14 bit. The pixel values depend on the integration time of the IR camera, the emissivity of the DUT surface and the temperature of the device. To determine the temperature of the device, a calibration of the raw data values is required [8].

First, an evaluation is performed at a constant ambient temperature $\left(30^{\circ} \mathrm{C}\right)$ with 35 infrared images taken at different focus positions. The device T26 is used for this evaluation. Infrared images are taken at different positions on the optical axis in the range of $-44.41 \mathrm{~mm}$ to $-44.55 \mathrm{~mm}$. 
Visual inspection shows that a distinction between the focus image and blurred images is difficult (see figure 5). Three images (c.f. figure 5.b - 5.d) appear sharp by subjective visual impression. These images and the corresponding positions are chosen as references. However, infrared images which are more than $\pm 30 \mu \mathrm{m}$ away from the center position $-44.51 \mathrm{~mm}$ are even under subjective visual inspection recognised as blurred images (cf. figure 5.a and 5.e).

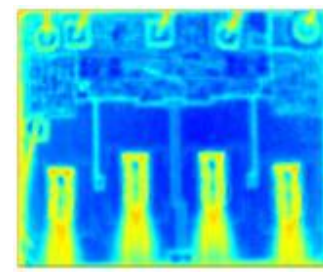

a) Pos. $-44.41 \mathrm{~mm}$

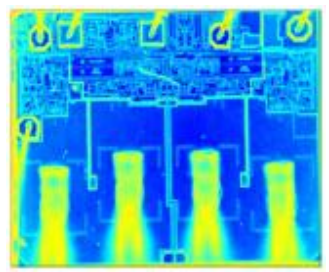

b) Pos. $-44.50 \mathrm{~mm}$

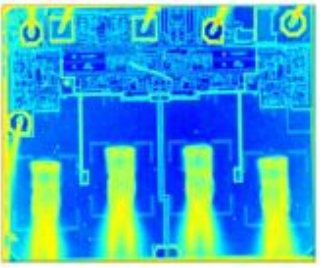

c) Pos. $-44.51 \mathrm{~mm}$

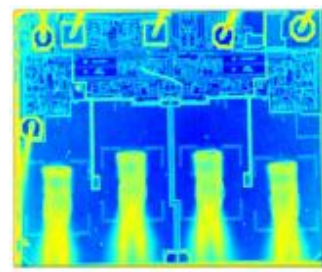

d) Pos. $-44.52 \mathrm{~mm}$

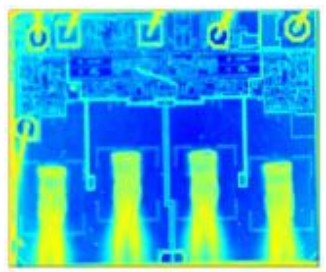

e) Pos. $-44.55 \mathrm{~mm}$

Fig. 5. Example for visual distinction of infrared images at different focus positions over a range of 140 um (false color images)

\subsection{Comparison of the auto focus function at ambient temperature}

In the next step, all three presented functions are analyzed and evaluated at ambient temperature. The Sobel edge detection, 2D FFT and the gradient operator are applied to the 35 images taken at different positions in direction of the optical axis. The results are illustrated in figure 6 to figure 8 . The focus value $\left(Y_{F}\right)$ for each curve is normalized between 0 and 1 for a better representation.

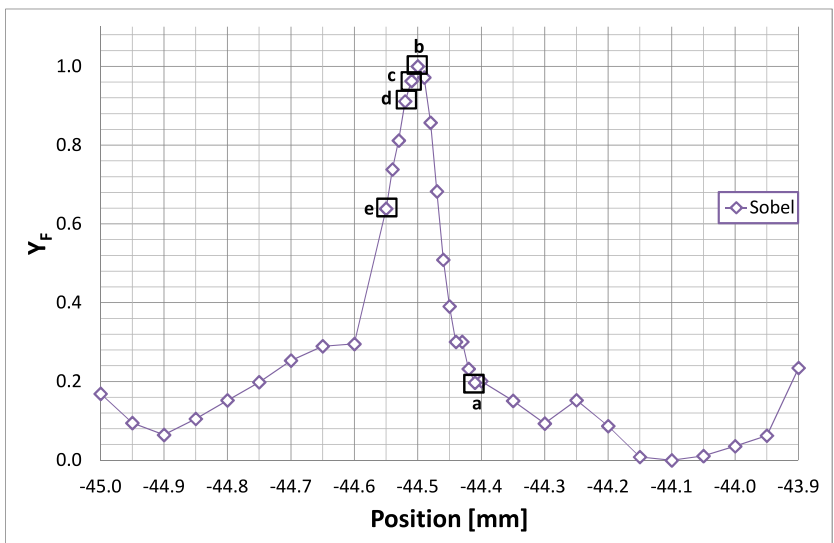

Fig. 6. Focus curve using the Sobel edge function. The letters correlate to images of figure 5.

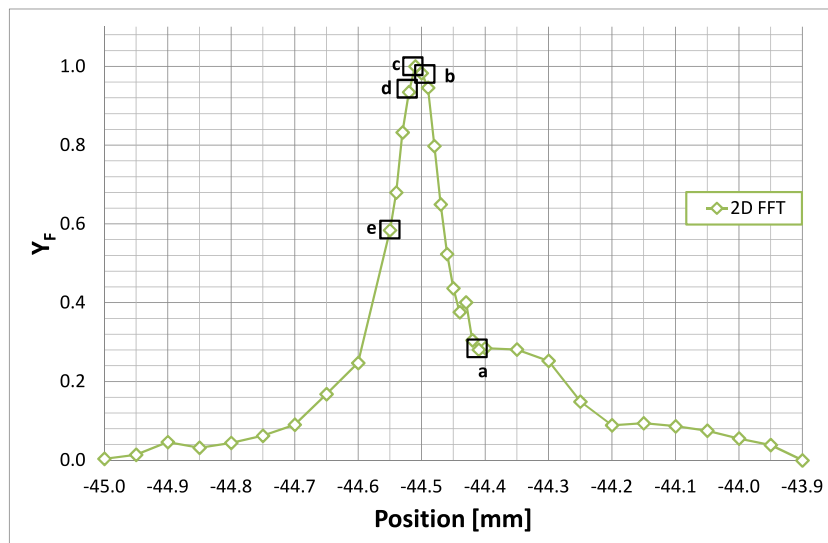

Fig. 7. Focus curve using the 2D FFT method. The letters correlate to images of figure 5.

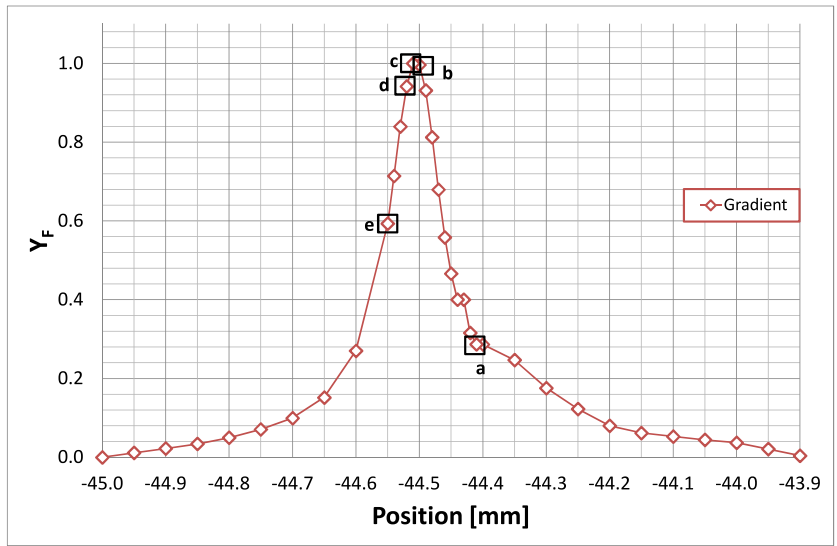

Fig. 8. Focus curve using the gradient method. The letters correlate to images of figure 5.

Each focus curve is evaluated based on the criteria listed in [9]. The criteria describe that the focus curve should have only one maximum and no further maxima (Unimodality). An optimal reproducibility can be guaranteed when the focus curve has a sharp peak (Reproducibility). The expected focus image should correlate exactly to the maximum of 
the focus curve (Accuracy). There should be no limitation to device structures that may be focused (General applicability).

All focus curves fulfill the selected criteria and all detected focus images lie in the expected focus range. The general applicability has been verified with all three different test devices. In the context of computation time our evaluation showed that the edge detection is three times slower than the other two methods. The edge detection uses the gradient operator and a threshold function to detect the edges, hence the algorithm requires more computation time. However, computation time strongly depends on how the respective functions are implemented and optimized in Matlab, and hence a comparison of computation speed is of limited significance. We can only conclude that edge detection is too slow regarding our particular application and implementation.

\subsection{Comparison of the auto focus function at different temperatures}

The auto focus functions can detect the focus image and the corresponding focus position at different temperatures. A test set of infrared images taken at different stable temperatures is generated. First the Sobel edge detection is executed on the infrared image sequence. Although the function shows adequate results for detecting the focus image at ambient temperature, the focus curve shows multiple maxima (c.f. figure 9) for various stable temperatures.

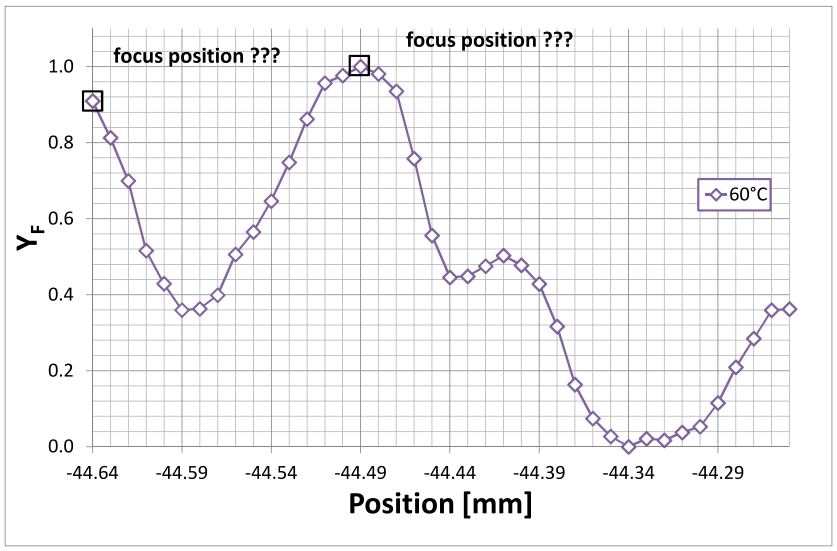

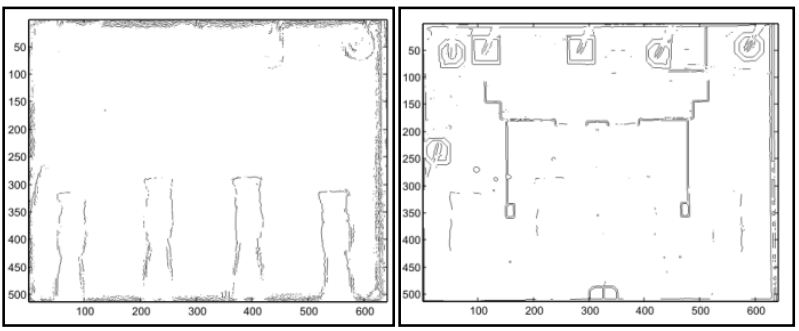

a) blurred image

b) focus image

Fig. 10. Visual inspection of both maxima of the focus curve represented in figure 9

Fig. 9. Edge detection result at $60^{\circ} \mathrm{C}$

The visual inspection of figure 10 shows that the "blurred" image has a lot of edges at the border and the bond wire outlines can be seen (c.f. figure 4). In our case another focus plane is actually detected, more precisely that of the bond wires. Consequently, the focus curve contains multiple maxima. Thus, the Sobel edge operator is rejected, because on the one side it is not robust enough against noise at higher temperatures and on the other side it does not detect the desired focus position.

The gradient operator and the 2D FFT have a unique global maximum in their focus curve (c.f. figure 11). At stable temperature the focus image and corresponding focus position can be detected in all cases. Each curve has a similar behavior. A shift of the focal plane is caused by thermo-mechanical expansion of the DUT mounting stage as well as the device itself during the heating up process.

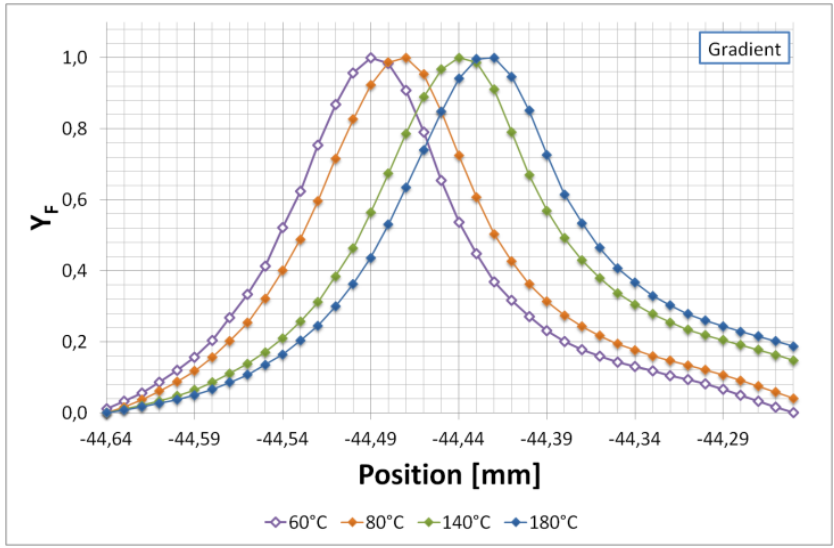

a) Gradient operator

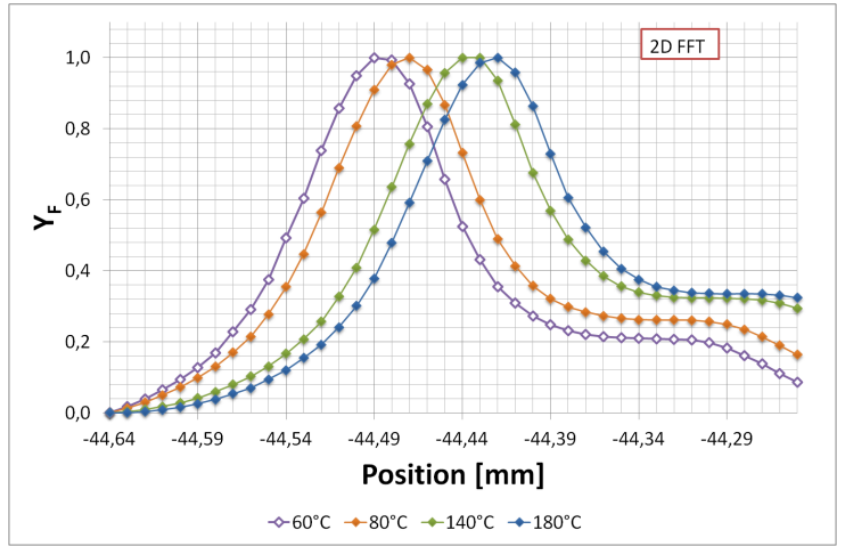

b) 2D FFT operator

Fig. 11. Focus curves at different stable temperatures 
The 2D FFT and the gradient operator both deliver consistent results. Although the 2D FFT showed a marginally better performance related to computation time especially in Matlab, the mathematically simpler gradient method is selected for our final implementation. Therefore, further evaluations within this paper apply only the gradient operator.

\subsection{Investigation of infrared images taken at changing temperatures}

The illustrated and evaluated algorithms - excluding the Sobel edge detection - work well for stable temperatures. This means that during the whole search process the temperature does not change for more than $\pm 2{ }^{\circ} \mathrm{C}$. During a heating up process or cooling down phase of a device the camera goes gradually out of focus. An algorithm is required that can follow the focus image in real time. The challenge is that infrared images have different raw data distributions at different temperatures. The change in raw data over temperature can be explained by several effects, like electrical and optical camera bandwidth, temperature-dependent emission (i.e. Planck's law) and sample surface emissivity. Figure 12 shows the raw data distribution of an infrared image taken at $80^{\circ} \mathrm{C}$ and $180^{\circ} \mathrm{C}$. The raw data distribution at $80^{\circ} \mathrm{C}$ is in a range of 3240 to 3400 , whereas the raw data distribution at $180^{\circ} \mathrm{C}$ is in a range of 3370 to 4370. In figure 13 the corresponding images of the histograms are illustrated. Both images are scaled to the same palette (see color bar), hence the image taken at $80^{\circ} \mathrm{C}$ looks significantly darker.

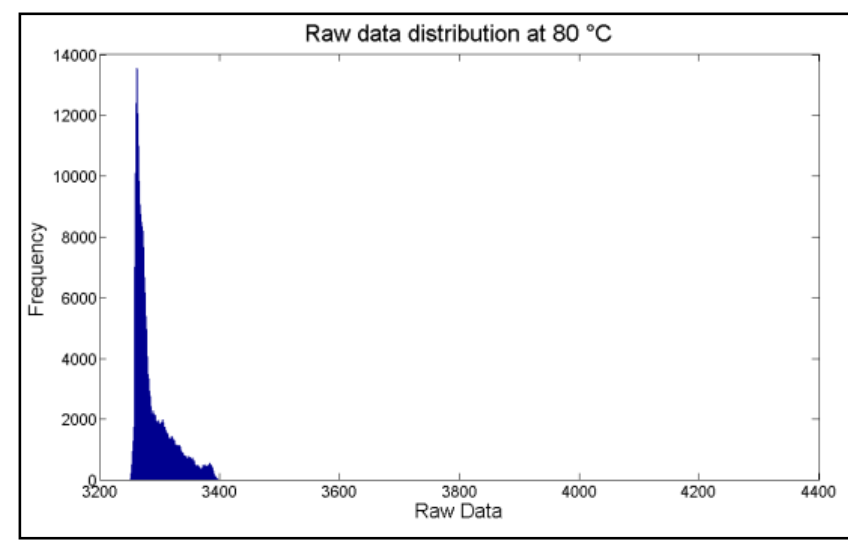

a) Raw data distribution at $80^{\circ} \mathrm{C}$

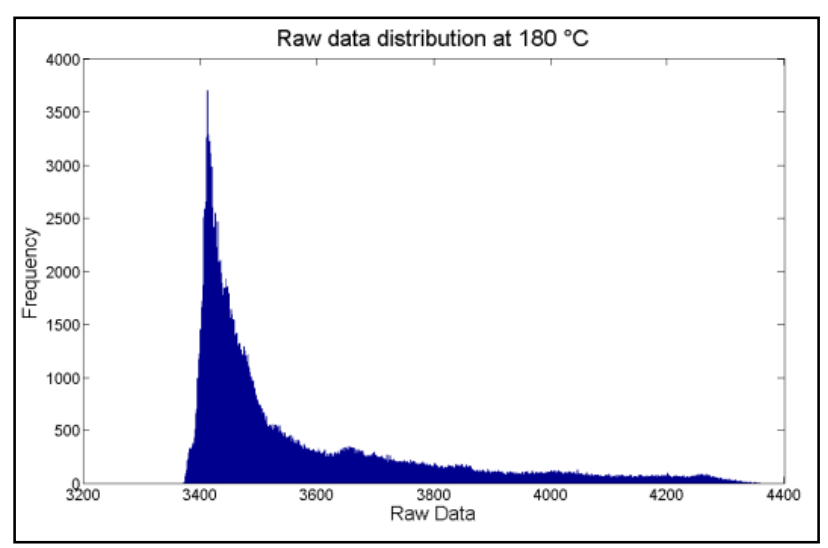

a) Raw data distribution at $180^{\circ} \mathrm{C}$

Fig. 12. Histogram of device T26 at different temperatures

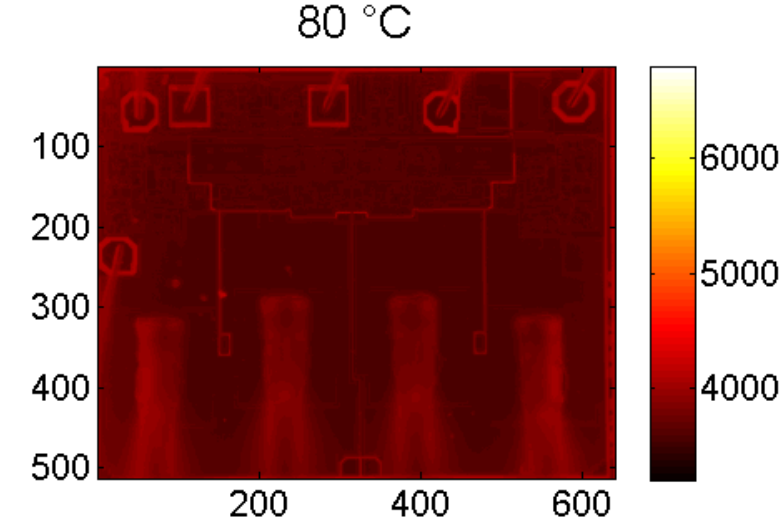

a) IR image at $80^{\circ} \mathrm{C}$

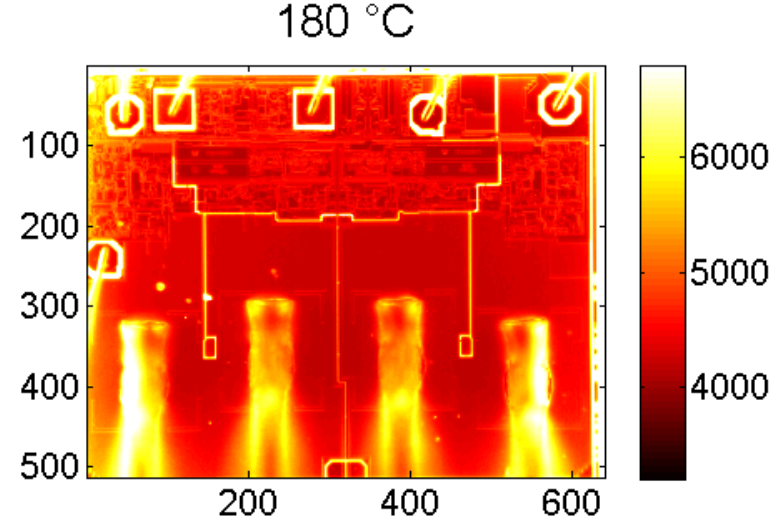

b) IR image at $180^{\circ} \mathrm{C}$

Fig. 13. IR intensity images of a power semiconductor at different temperatures, higher intensities at higher temperatures. (Same scale and false color images)

We initially assumed that the defocusing has more influence on the focus value $Y_{F}$ than the temperature change (c.f. figure 14). Thus, a shift in the optical axis might be detected unambiguously. However, the measurements and the evaluation showed that the differences in the raw data distribution have a higher influence than the defocusing. The focus value increases with rising temperatures, but decreases due to defocusing (c.f. figure 14 and figure 15). 


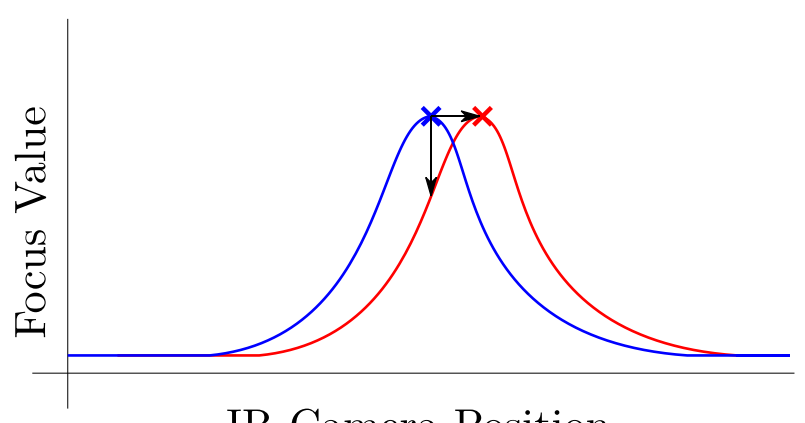

IR Camera Position

a) Expected behavior of the focus curve

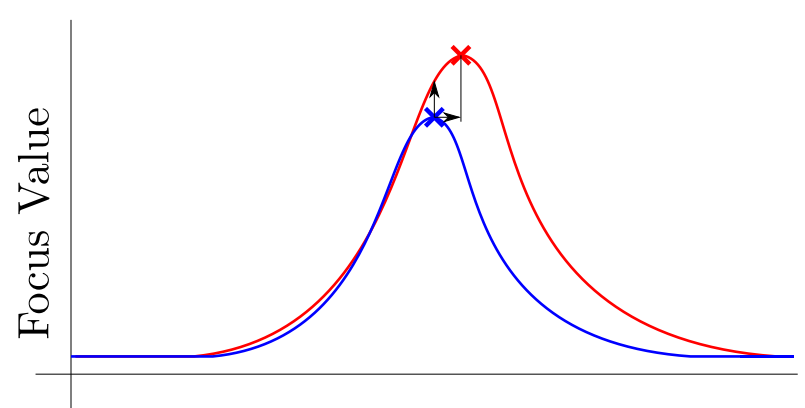

IR Camera Position

b) Detected behavior of the focus curve

Fig. 14. Behavior of the focus curve during heating up process

If the focus curves of figure 11.a are not individually normalized between 0 and 1 for each curve, but normalized between 0 and 1 for the total maximum and minimum of all curves, the global rising of the focus value $Y_{F}$ can be seen (c.f. figure 15). Following observations are evaluated based on figure 15. The first observation shows that the focus position shifts along the optical axis. This can be explained by the thermal-mechanical expansion of the DUT. The second observation says that the focus direction is not changing consistently in the same direction. This is caused by asymmetries in the mechanical mounting of the DUT on the DUT stages.

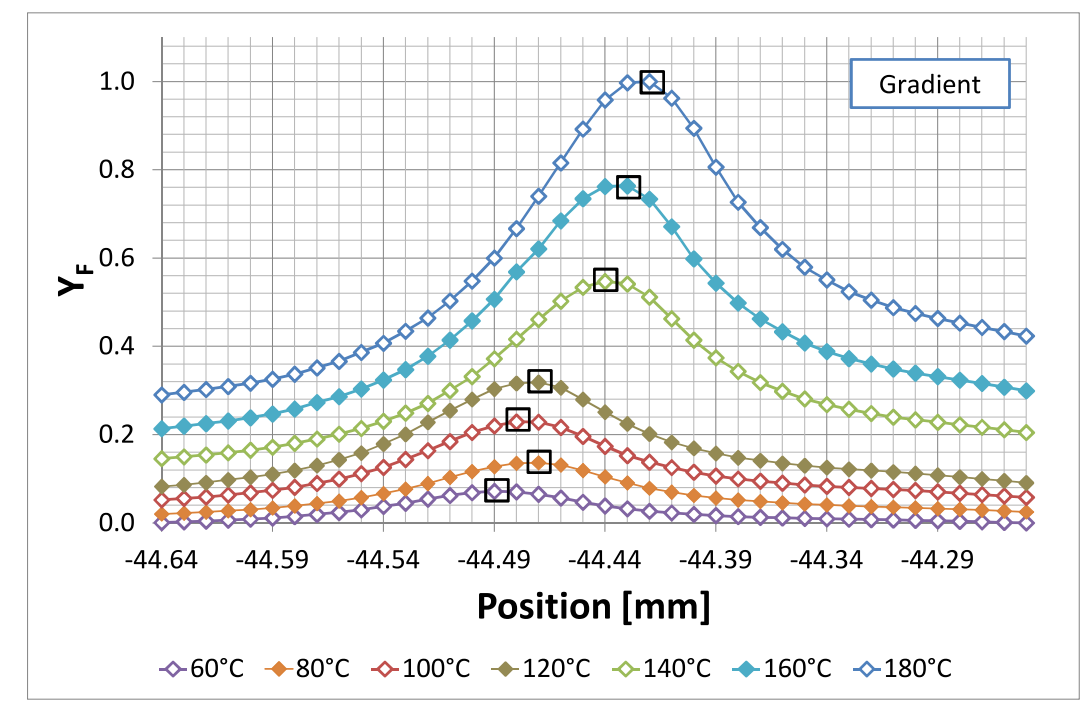

Fig.15. Focus curve at different temperatures using the gradient method. The focus value increases with rising temperature.

During the real time auto focusing, the temperature of the device and the focus direction are unknown. One possible solution to solve this challenge is to make sure that the change between two focus values $\Delta \mathrm{Y}_{1,2}$ regarding the temperature change $\Delta \mathrm{T}$ is lower than the change between two focus values $\Delta \mathrm{Y}_{1,2}$ regarding the position $\Delta \mathrm{P}$. This means equation 2 has to be fulfilled.

$$
\frac{\Delta \Upsilon_{1,2}}{\Delta \mathrm{T}} \leq \frac{\Delta \mathrm{Y}_{1,2}}{\Delta \mathrm{P}}
$$

If the rise of the focus curve due to temperature change is faster than the focus value shift due to defocusing, another approach will be required. We are working on a physical focus prediction model, where the temperature and the focus values are estimated out of the raw data history. A mathematical estimation model may be able to detect the focus value even for rapid temperature changes. The basic challenge is to generate a physical model that can operate independently of the test device surface structure.

\section{Conclusion}

We presented a real time auto focus for infrared imaging at stable and slowly changing temperatures. Three different auto focus functions were implemented and experimentally evaluated. The gradient and 2D FFT methods show satisfactory results. Both algorithms represent a single and clear maximum in the focus curve, can handle intensity 
changes and have a good performance for the real time implementation. The edge detection shows several local maxima in the focus curve and might be misled by ambiguous focus positions, e.g. the bond wires. Thus, this method is not useful for detecting the focus image in our application. For the sake of simplicity, we use the gradient operator for our final implementation.

To detect the focus image at changing temperature the device needs to be heated up slowly such that the change of defocusing still remains the dominant parameter. Focusing methods for this quasi-static case were proposed in the current paper and the limits of this approach were determined. A more complex concept based on a physical model for rapidly changing temperature and focus will be introduced in a future publication.

\section{Acknowledgment}

The authors would like to thank Mrs. Olivia Bluder and Mrs. Kathrin Plankensteiner for valuable discussions and mathematical and statistical support. Special thanks to Stefano de Filippis for his support with the technical camera setup.

This work was jointly funded by the Federal Ministry of Economics and Labour of the Republic of Austria (contract 98.362/0112-C1/10/2005 and the Carinthian Economic Promotion Fund (KWF) (contract 18911 | 13628 | 19100)

\section{REFERENCES}

[1] Geusebroek J. M., Cornelissen F., Smeulders A. W. M., Geerts H., "Robust autofocusing in microscopy", Cytometry, vol. 39, pp. 1-9, 2000.

[2] Wu Q., Merchant F. and K. Castleman, "Microscope Image Processing”, Academic Press, 1. edition, 2008.

[3] De Filippis S., "Development of an experimental setup for Infrared Camera characterization aimed at Smart Power devices Thermography," Master's thesis, Università degli Studi di Napoli Ferderico II, 2008.

[4] Einspieler S., "Development of a LabVIEW-based position control for an infrared thermal imaging system," Bachelor's thesis, Carinthia University of Applied Science Systems Engineering Course, 2010.

[5] Sobel I.E., "Camera models and machine perception", PhD thesis, Stanford, USA, 1970.

[6] Gonzalez R. C. and Woods R. E., "Digital Image Processing (3rd Edition)" Prentice-Hall, Inc., Upper Saddle River, USA, 2006

[7] Glavanovics M., Estl H. and Bachofner A., "Reliable smart power system ICs for automotive and industrial applications - the Infineon smart multichannel switch family", Proceedings of PCIM Europe, pp. 1-6, Nuremberg (Germany), 2001.

[8] Köck H., De Filippis S., Djelassi C., Kreuter H. P., Illing R., Glavanovics M., and Kaltenbacher M., "In-situ calibration procedure for infrared microscopy temperature measurements, "Proceedings of the Microtechnology and Thermal Problems in Electronics, pp. 1 - 6, 2011.

[9] Groen F. C., Young I. T. and Ligthart G., "A comparison of different focus functions for use in autofocus algorithms", Cytometry, vol. 6(2), pp. 81-91,1985. 